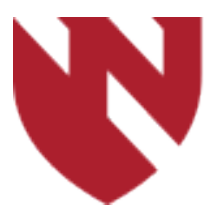

December 2019

\title{
National Trends in Survival for Pancreatic Adenocarcinoma Based on Location of the Primary Lesion
}

\author{
Gautam K. Malhotra \\ University of Nebraska Medical Center \\ Lynette M. Smith \\ University of Nebraska Medical Center \\ Quan P. Ly \\ University of Nebraska Medical Center \\ Aaron R. Sasson \\ University of Nebraska Medical Center \\ Chandrakanth Are \\ University of Nebraska Medical Center
}

Tell us how you used this information in this short survey.

Follow this and additional works at: https://digitalcommons.unmc.edu/gmerj

Part of the Higher Education Commons, and the Medicine and Health Sciences Commons

\section{Recommended Citation}

Malhotra, G. K., Smith, L. M., Ly, Q. P., Sasson, A. R., , Are, C. National Trends in Survival for Pancreatic Adenocarcinoma Based on Location of the Primary Lesion. Graduate Medical Education Research Journal. 2019 Dec 13; 1(1).

https://digitalcommons.unmc.edu/gmerj/vol1/iss1/44

This Conference Proceeding is brought to you for free and open access by DigitalCommons@UNMC. It has been accepted for inclusion in Graduate Medical Education Research Journal by an authorized editor of DigitalCommons@UNMC.For more information, please contact digitalcommons@unmc.edu. 
National Trends in Survival for Pancreatic Adenocarcinoma Based on Location of the Primary Lesion

\section{Creative Commons License}

\section{(c) (1) $\Theta(9$}

This work is licensed under a Creative Commons Attribution-Noncommercial-No Derivative Works 4.0 License. 
to be weaned off hemodialysis along with the benefit of concurrent amelioration of renovascular hypertension.

Conclusion: For select patients with RAO there can benefits, such as achieving dialysis independence and stabilization of renovascular hypertension, by attaining revascularization of the renal arteries despite prolonged time of ischemia.

https://doi.org/10.32873/unmc.dc.gmerj.1.1.041

\section{No Survival Advantage Exists for Patients Undergoing Loop lleostomy for Clostridium difficile Colitis Bradley R. Hall, Andrew Cannon, Pranita Atri, Christopher S. Wichman, Lynette M. Smith, Apar K. Ganti, Chandrakanth Are, Aaron R. Sasson, Sushil Kumar, Surinder K. Batra}

\section{Mentor: Dmitry Oleynikov \\ Program: General Surgery}

Background \& Objectives: Loop ileostomy (LI) remains an unproved alternative to total abdominal colectomy (TAC) for patients with Clostridium difficile infection (CDI). We aim to compare outcomes between loop ileostomy and total abdominal colectomy for Clostridium difficile infection using a national database.

Methods: The 2011-2016 ACS-NSQIP database was queried for patients who underwent LI or TAC for CDI. Patients with high outlying age, LOS, and operative time were excluded. Statistics were performed using IBM SPSS and NCSS PASS 11.

Results: Of 457 patients identified, 47 underwent LI. Predicted morbidity was higher in the TAC cohort $(62 \%$ vs. $37 \%, \mathrm{p}<0.001)$. Patients in the LI cohort experienced fewer complications $(72 \%$ vs. $87 \%, \mathrm{p}=0.021)$; however, mortality did not differ between LI (36\%) and TAC (31\%) cohorts. Blood transfusions were more than twice as frequent in the TAC cohort ( $54 \%$ vs. $19 \%$, $\mathrm{p}<0.001)$. Four patients in the LI cohort required reoperation; however, none required colectomy.

Conclusions: Compared to TAC, LI does not improve survival for patients who require surgical intervention for CDI.

https://doi.org/10.32873/unmc.dc.gmerj.1.1.042

\section{Advanced Pancreatic Cancer: A Meta-Analysis of Clinical Trials over Thirty Years Bradley R. Hall, Jennifer A. Leinicke, Priscila R. Armijo, Lynette M Smith, Sean J. Langenfeld, Dmitry Oleynikov}

Mentor: Surinder Batra

Program: General Surgery

Background \& Objectives: In contrast to other cancers, survival rates for pancreatic ductal adenocarcinoma (PDAC) have improved but minimally over the past thirty years. The aim of this study was to perform a meta-analysis of clinical trials published since 1986 to determine trends in median overall survival in primarily metastatic PDAC.

Methods: All Phase 2-4 clinical trials published during or after 1986 investigating first-line systemic chemotherapy in metastatic PDAC were included in the meta-analysis. Publications obtained through PubMed and
www.ClinicalTrials.gov were cross-referenced to identify additional trials. Trials enrolling fewer than $50 \%$ of study participants with metastatic disease were excluded.

Results: Of 19,488 patients enrolled in 151 clinical trials, $84 \%$ had metastatic disease and $16 \%$ had locally advanced pancreatic cancer. In clinical trials published from 1986 to 2016 , the weighted median overall survival (wMOS) increased by 3.0 months. The median wMOS was higher in combination therapy (7.31 months, IQR 5.4 to 8.5) compared to non-gemcitabine, single-agent therapy (4.76 months, IQR 3.5 to 6.0), gemcitabine monotherapy (6.48 months, IQR
5.9 to 7.2 ), and gemcitabine plus single-agent therapy (7.09 months, IQR 6.3 to 8.2). Of all regimens used in more than one study arm, FOLFIRINOX had the highest wMOS (10.9 months).

Conclusions: Regardless of treatment regimen, survival rates in PDAC have minimally improved over time. Of drugs used in two or more study arms, only FOLFIRINOX has a wMOS greater than ten months. Emphasis should, therefore, be placed on identification of novel targets that promote early diagnosis and intervention.

https://doi.org/10.32873/unmc.dc.gmerj.1.1.043

\section{National Trends in Survival for Pancreatic Adenocarcinoma Based on Location of the Primary Lesion Gautam K. Malhotra, Lynette M. Smith, Quan P. Ly, Aaron R. Sasson, Chandrakanth Are}

Mentor: Chandrakanth Are

Program: General Surgery

Background: The management and prognosis of pancreatic adenocarcinoma varies depending on the location of the primary lesion within the pancreas. There is a paucity of data on survival trends in pancreatic adenocarcinoma based on the location. The aim of this study was to analyze the national trends in survival for patients with pancreatic adenocarcinoma based on the location of the primary lesion.

Methods: The Surveillance, Epidemiology, and End Results (SEER) database (SEER 17, 1973-2007) was used to identify patients with pancreatic cancer located in the head (C25.0), body (C25.1), or tail (C25.2). SEER*Stat
6.6.2 was used to calculate 5-year survival data. To stabilize rates, we used 5-year cohorts when calculating trends.

Results: A total of 89,733 patients were included in the study. The overall 5-year survival, inclusive of all locations and treatment approaches was is $5.4 \%(95 \% \mathrm{CI}$ 5.2-5.6\%). For lesions in the head, body and tail the overall 5-year survival, inclusive of 
all treatment approaches, was 5.2\% (95\% CI 5-5.5\%), 3.9\% (95\% CI 3.4-4.4\%) and 7.8\% $(95 \%$ CI $7.2-8.5 \%)$ respectively. For patients that underwent surgery, the 5-year survival for patients with lesions in all locations, head, body and tail was $18.8 \%, 15.9 \%, 23.8 \%$ and $33.8 \%$ respectively. When analyzing trends we noted a significant improvement in survival for patients that underwent operative intervention. Comparing the time periods from 1977-1981 versus 2002-2006, the 5-year survival for surgical patients improved for all locations (9.2\% to $22.6 \%, \mathrm{p}<0.05)$ and also for lesions in the head $(8.3 \%$ to $19.3 \%$, $\mathrm{p}<0.05)$, body $(6.7 \%$ to $26 \%, \mathrm{p}<0.05)$ and tail $(14.7 \%$ to $40.7 \%, \mathrm{p}<0.05)$.

Conclusion: The results of our study demonstrate an improvement in survival for

\section{Global Trends in Esophageal Cancer}

Gautam K. Malhotra, Ujwal Yanal, Advaitaa Ravipati, Matthew Follet, M. Vijayakumar, Chandrakanth Are

\section{Mentor: Chandrakanth Are \\ Program: General Surgery}

Background \& Objectives: Esophageal Cancer (EC) is a lethal malignancy with poor prognosis and significant variations in the incidence, mortality, and histopathology based on geographic regions. The aim of this study was to quantitatively analyze these variations to identify patterns and areas for further research.

Methods: We utilized the GLOBOCAN 2012, and Cancer Incidence in five Continents,
Volume X (CI5X) database to analyze variations in $\mathrm{EC}$ incidence and mortality.

Results: We found the EC incidence and mortality is geographically varied with a particularly high burden in East Asia and Eastern/Southern Africa where esophageal squamous cell carcinoma (SCC) predominates over adenocarcinoma (AC). Interestingly, there is a dichotomy between the high incidence of esophageal SCC in East Africa and low incidence in West Africa. The global incidence and mortality from $\mathrm{EC}$ is expected to rise in the coming decades. Asia, and patients with pancreatic cancer treated by operative intervention. This improvement in survival was more pronounced for patients with primary lesions located in the body and tail.

https://doi.org/10.32873/unmc.dc.gmerj.1.1.044

\section{Outcomes of Intragastric Balloon Placements: An Analysis of the 2016 MBSAQIP Database \\ Bhavani Pokala, Priscila Armijo, Dmitry Oleynikov}

Mentor: Dmitry Oleynikov

Program: General Surgery

Background \& Objectives: Intragastric balloon placement is an approved weightloss procedure with a lower BMI cutoff than bariatric surgery. The objective of this study was to examine the frequency and outcomes of balloon placements in 2016 .

Methods: The MBSAQIP database was analyzed for adults who underwent elective, endoscopic intragastric balloon placement. Emergent cases, patients with previous foregut surgery and those unable to be followed for 30 days were excluded. Comparisons were made between placements performed by GI and surgery. Analysis performed using SPSS $\mathrm{v} 25.0, \alpha=.05$.

Results: Our study included 445 patients. Majority were female (80\%) and Caucasian (90.6\%). Median age and BMI were 49.0 (IQR 15.4) and 35.7 (IQR 6.46), respectively. Prevalence of medically treated hypertension was $30.8 \%$, hyperlipidemia $18.9 \%$, GERD $20.4 \%$, and diabetes $12.6 \%$ (insulin and non-insulin dependent). Mean operative time was $15.48 \pm 9.89$ minutes. No 30 -day mortalities were reported. 70 balloons were placed by GI and 375 by surgery. There was a significantly higher rate of readmission for GI $7.1 \%$ vs $1.9 \%$ surgery $(p=.027)$. No significant difference was seen for rates of post-operative
China in particular, will continue to be the areas most burdened by EC, while Africa is expected to surpass the incidence and mortality rates of Europe.

Conclusions: The global burden of EC is expected to rise in the coming years. Understanding the geographic, environmental, and genetic contributors to the development of EC will be essential in combating its prevalence.

https://doi.org/10.32873/unmc.dc.gmerj.1.1.045

\section{Development of a Porcine Model of Severe Noncompressible Truncal Hemorrhage \\ Ujwal Yanala, Jason Johanning, Iraklis Pipinos, William Velander, Mark Carlson}

\section{Mentor: Mark Carlson}

Program: General Surgery

Introduction: Noncompressible truncal hemorrhage and brain injury account for most early mortality in military personnel on the battlefield. Currently there is no effective treatment for noncompressible truncal hemorrhage, other than rapid evacuation to a surgical facility. We intended to develop a porcine model of noncompressible truncal hemorrhage so that we might be able to develop new treatments for this clinical problem.

Methods: Swine (boars, 3 months, $32-34 \mathrm{~kg}$ ) underwent one of three injury types: 1) central stellate injury, just anterior to suprahepatic inferior vena cava $(\mathrm{N}=6) ; 2$ ) excision of a portal vein branch distal to the main trunk $(\mathrm{N}=5)$; or 3 ) near-transection of the left lateral lobe (LLL) of the liver at its base, which dehydration (GI: 7.1\% vs surgery: $7.2 \%$; $\mathrm{p}=1.000$ ), emergency room encounters (GI $14.0 \%$ vs surgery: $4.0 \% ; p=.486), 30$-day reoperations (GI: $0.0 \%$ vs surgery: $0.8 \%$; $\mathrm{p}=1.000$ ), and 30-day reinterventions (GI: $5.7 \%$ vs surgery $2.4 \% ; \mathrm{p}=.132$ ).

Conclusions: The majority of patients undergoing intragastric balloon placement fall within obesity class 1 or 2 and have low rates of comorbidities. The procedure can be performed by gastroenterology or surgery at bariatric centers with similar outcomes. https://doi.org/10.32873/unmc.dc.gmerj.1.1.046 cut the hepatic and portal veins to this lobe $(\mathrm{N}=12)$. The midline incision was towel clipped immediately after injury, and animals were monitored for $60 \mathrm{~min}$ or until death. Resuscitation was performed with warm LR (max volume $=100 \mathrm{~mL} / \mathrm{kg}$ ), with a target MAP set at $80 \%$ of pre-injury MAP.

Results: The starting weight, MAP, hemoglobin, arterial $\mathrm{pH}$, INR, and pre-injury blood loss did not differ among groups $(p$ 